\title{
Towards constitutive equations for the deep Earth II: Shear properties under pressure
}

\author{
B.L.N. Kennett \\ Research School of Earth Sciences, The Australian National University, Canberra, ACT 2601, Australia
}

\section{A R T I C L E I N F O}

\section{Keywords:}

Constitutive equations

Equations of state

Bulk modulus

Shear modulus

Deep Earth

\begin{abstract}
A B S T R A C T
An equation of state can be converted into a full constitutive equation by adding to the strain energy a deviatoric component as a function of density change, which specifies incremental shear properties under compression. A suitable functional form for the shear term can be found from a semi-empirical linear relation between shear modulus, bulk modulus and pressure satisfied by experimental and computational results, and current Earth models. A general representation for the shear modulus behaviour can then be provided in terms of the zero pressure values of the shear and bulk modulus $\left(\mathrm{G}_{0}, \mathrm{~K}_{0}\right)$ and their pressure derivatives $\left(\mathrm{G}_{0}{ }^{\prime}, \mathrm{K}_{0}{ }^{\prime}\right)$. Shear modulus formulations are provided for equations of state in current use. The moduli for a third-order Birch-Murnaghan development diverge from the expected linear behaviour at high pressures. This divergence may help to explain problems encountered in fitting both shear and bulk modulus behaviour for the deep Earth using mineral physics models. Suitable functional forms for constitutive equations for the deep Earth need to take into account the expected behaviour at extreme pressure.
\end{abstract}

\section{Introduction}

A variety of equations of state are used for the fitting of experimental data for the bulk modulus $K$ at high pressures (e.g., Sakai et al., 2016), but only the Birch-Murnaghan formulation currently provides a direct link to the shear modulus G (Stixrude and Lithgow-Bertelloni, 2005). Increasing information on shear properties under pressure has been obtained in recent years. Melinger-Cohen and Jeanloz (2019) present results for the pressure dependence of both bulk and shear moduli for a range of materials.

In Kennett (2017) I introduced an approach that allows the association of a shear modulus with any equation of state specifying the bulk modulus as a function of volumetric strain. This is achieved by introducing an additional term into the strain energy in the form of a function of volumetric strain modulating a deviatoric component that vanishes in pure compression. This function is directly related to the shear modulus. The specification of the dependence of the shear modulus on change of volume, and hence pressure, is then based on an semiempirical linear relationship between the shear modulus, bulk modulus and pressure.

Here I consider the foundations of this linear relationship, which gives a good representation of experimental and computational results at high pressure, and is satisfied well by radial Earth models (e.g., $a k 135$, Kennett et al., 1995). The linear relation provides a general representation for the shear modulus in terms of the zero pressure values of the shear and bulk modulus $\left(\mathrm{G}_{0}, \mathrm{~K}_{0}\right)$ and their pressure derivatives $\left(\mathrm{G}_{0}{ }^{\prime}, \mathrm{K}_{0}{ }^{\prime}\right)$. This enables a functional form for the shear modulus to be specified for any equation of state (EOS). Explicit forms for the shear modulus are provided for a number of formulations in the Appendix. The generation of explicit forms for the shear modulus enlarges the repertoire of available ways of describing material behaviour under high pressure.

The limit of infinite pressure is frequently invoked to introduce constraints on the forms of equations of state, and so I also consider the way in which this limit can be exploited for shear properties.

\section{Shear effects under pressure}

For the application of the Gibbs free energy treatment of phase equilibria for multi-component systems (Stixrude and LithgowBertelloni, 2011), each component must be specified in terms of a strain energy function that can be identified with the Helmholtz free energy. This imposes significant constraints on acceptable forms for constitutive equations.

Kennett (2017) has cast the strain energy function $W$ for an isotropic medium, as a function of strain invariants, in a form that allows complete separation between hydrostatic and deviatoric components. The strain energy formulation is most naturally expressed in terms of

E-mail address: Brian.Kennett@anu.edu.au. 


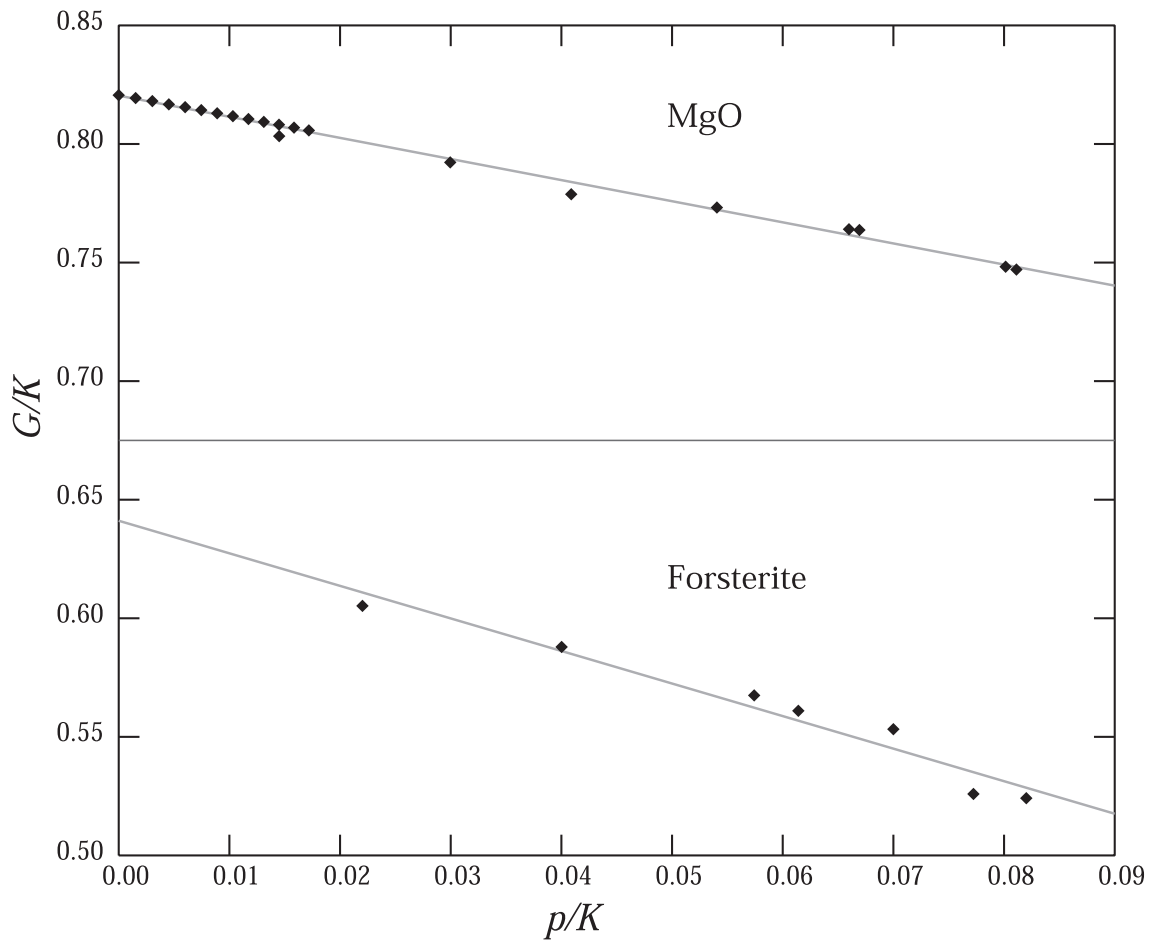

Fig. 1. Illustration of the linear dependence of $G / K$ on $p /$ $\mathrm{K}$ to $20 \mathrm{GPa}$ pressure, for the adiabatic bulk modulus $\mathrm{K}$ : Upper panel: periclase (MgO) using data from Jackson and Niesler (1982); Sinogeikin and Bass (2000); Zha et al. (2000). Lower panel: forsterite $\left(\mathrm{Mg}_{2} \mathrm{SiO}_{4}\right)$, the magnesium rich end member of the olivine solid solution series, from the results of Zha et al. (1996). volumetric strain, with pressure as a derived quantity. As such it is well suited to the direct analysis of synchrotron experiments in which volume is directly estimated and the problems of pressure calibration is avoided (e.g., Kono et al., 2010).

In terms of the initial density $\rho_{0}$ and the deformed density $\rho$, we introduce the volumetric strain $J=\rho_{0} / \rho$. The strain energy can then be written as

$\mathrm{W}=\Phi(\mathrm{J})+\{\mathrm{L}-3\} \Psi(\mathrm{J})$,

where the second invariant of strain $\mathrm{L}$ is normalised so that the deviatoric term $\{\mathrm{L}-3\}$ vanishes for hydrostatic deformation (Kennett, 2017). For small strains about a hydrostatic state, the pressure p, bulk modulus $\mathrm{K}$ and shear modulus $\mathrm{G}$ can be related directly to the functions of volumetric strain:

$\mathrm{p}=-\frac{\partial \Phi}{\partial \mathrm{J}}, \quad \mathrm{K}=\mathrm{J} \frac{\partial^{2} \Phi}{\partial \mathrm{J}^{2}}, \quad G=\frac{1}{\mathrm{~J}} \frac{\partial \Psi}{\partial \mathrm{J}}$.

This approach allows the retention of familiar forms for equations of state specified by the behaviour of $\Phi(J)$ with the addition of a full description of shear through $\Psi(\mathrm{J})$. In principle, this formulation provides very considerable flexibility in representation, but there remains the issue of relating the two functions $\Phi(\mathrm{J}), \Psi(\mathrm{J})$. Derivatives such as $\mathrm{G}^{\prime}=\partial \mathrm{G} / \partial \mathrm{p}$ intrinsically couple the dependence on $\Phi(\mathrm{J})$ and $\Psi(\mathrm{J})$.

The equation of state $\Phi(\mathrm{J})$ has been specified as a function of density (volume) through $\mathrm{J}$, which is an invariant of strain. Many developments for strain energy work with just quadratic strain, but a broad range of strain measures can be employed (e.g. Katsura and Tange, 2019). Deformation can be described by a stretch matrix $\mathrm{U}$ with principal stretches $\left\{\lambda_{\mathrm{i}}\right\}$ that will be equal for hydrostatic compression. The invariant $\mathrm{J}=\operatorname{det} \mathbf{U}$. The Seth-Hill class of strain measures (Seth, 1964; Hill, 1968) take the form:

$\mathbf{E}_{\mathrm{q}}(\mathbf{U})= \begin{cases}\frac{1}{\mathrm{q}}\left(\mathbf{U}^{\mathrm{q}}-\mathbf{I}\right) & \text { if } \mathrm{q} \neq 0, \\ \ln \mathbf{U} & \text { if } \mathrm{q}=0,\end{cases}$

where $\mathbf{I}$ is the identity tensor. All the members of this class of strain measures take the same form for infinitesimal deformation. The Lagrangian (Green) strain is thus $\mathbf{E}_{2}$, and the Eulerian $\mathbf{E}_{-2}$. The case $q=0$ corresponds to the Hencky strain used by Poirier and Tarantola (1998). For compression, the behaviour is best represented using measures depending on strain exponent $\mathrm{q}<0$, as in the standard Eulerian BirchMurnaghan representation, but in tension $\mathrm{q}>0$ is to be preferred (Beex, 2019). For $\mathrm{q}>0$ the pressure does not approach $\infty$ when the volume tends to zero. This is why the Eulerian Birch-Murnaghan system provides a much better representation of experimental results than the Lagrangian (Melinger-Cohen and Jeanloz, 2019).

We can expect that the dependence of the functions $\Phi(\mathrm{J})$ and $\Psi(\mathrm{J})$ on volume change $J$ should be somewhat similar. Insight into the nature of links between the moduli for crystalline solids can be obtained from lattice dynamics. The acoustic modes of vibration depend on the elastic moduli (e.g., Kieffer, 1979). In order to obtain a full representation of the behaviour as a function of wave vector across the full Brillouin zone, the interactions between a range of nearby atoms have to be considered. For example, Zhang et al. (2011) found that they needed to include at least 4-atom interactions for silicon. Stretching forces constants are normally much larger than bond bending constants, e.g., Elcombe (1967) suggests a factor of 8 for $\alpha$-quartz. Under compression, both stretching and bending contributions act in consort, but in shear the bending terms counteract the stretching (e.g., Zhang et al., 2011). For a crystal under pressure, Falzone and Stacey (1980) have used a model with a purely central potential function with changes in bond lengths between atoms in the deformed crystal calculated to secondorder in strain. This simplified model produces a linear relation between the shear modulus $\mathrm{G}$, bulk modulus $\mathrm{K}$ and pressure $p$ of the form

$\mathrm{G}=\mathrm{aK}-\mathrm{bp}$

This relation is consistent with the interactions of more general stretching and bending contributions under pressure, and is satisfied very well by experimental results on many different classes of minerals. For example, we show in Fig. 1 plots of $\mathrm{G} / \mathrm{K}$ against $\mathrm{p} / \mathrm{K}$ for both $\mathrm{MgO}$ and forsterite that show a well defined linear dependence. In Fig. 2 we show an extended range of pressure with theoretical estimates from the work of Karki et al. (1997) for $\mathrm{MgO}$, and experimental values for an iron bearing ferropericlase (Marquardt et al., 2009) for pressures below the iron spin transition. The theoretical shear modulus estimates from Karki et al. (1997), show variations due to changing anisotropy of the $\mathrm{MgO}$ 


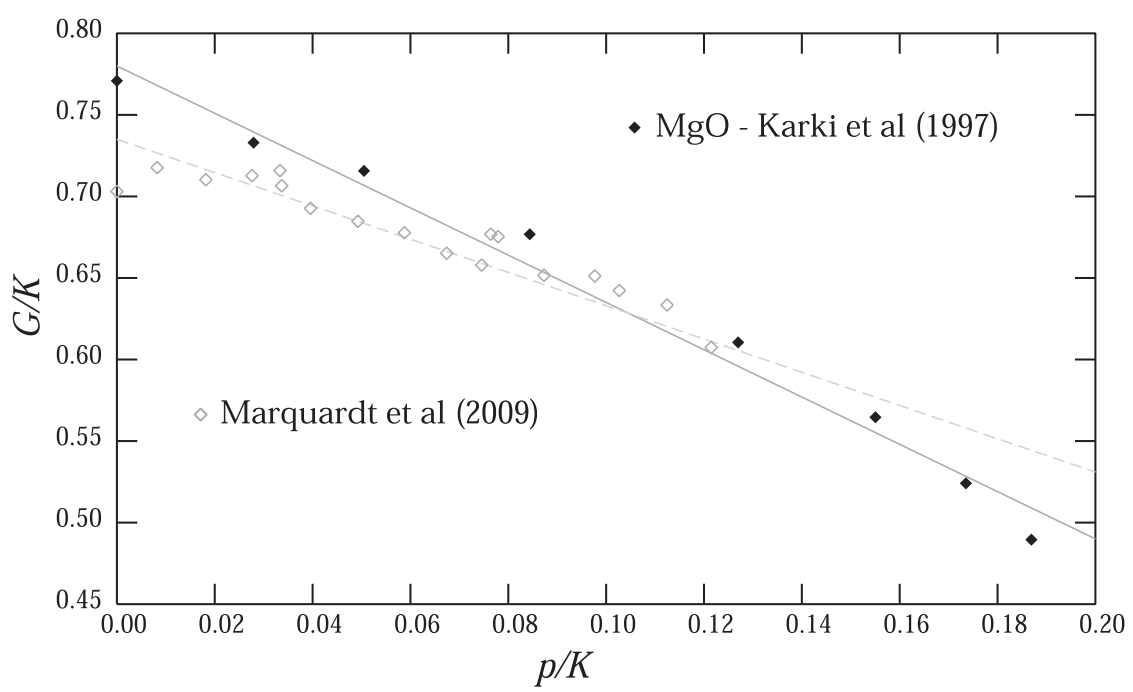

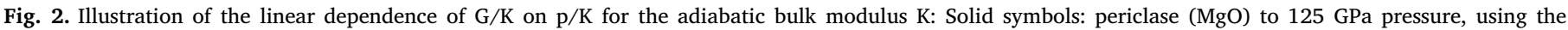
theoretical results of Karki et al. (1997). Open symbols: ferropericlase $\left(\mathrm{Mg}_{0.9} \mathrm{Fe}_{0.1} \mathrm{O}\right.$ ) to $40 \mathrm{GPa}$ from the results of Marquardt et al. (2009).

crystal, and lie well below the Brillouin scattering results of Murakami et al. (2009). Once again the linear relation provides a reasonable representation of the behaviour.

Kennett (2017) - Fig. 1 - has shown that the same linear dependence applies to the shear and bulk moduli as a function of pressure for the radial Earth model ak135 (Kennett et al., 1995; Montagner and Kennett, 1996), and a similar behaviour is found for PREM (Dziewoński and Anderson, 1981). In the travel time model ak135, the P and S wavespeed distributions are determined separately. The bulk and shear modulus are derived from

$K=\rho\left[\alpha^{2}-\frac{4}{3} \beta^{2}\right], \quad G=\rho \beta^{2}$,

in terms of the density $\rho, \mathrm{P}$ wavespeed $\alpha$, and shear wavespeed $\beta$. The pressure $\mathrm{p}$ is derived from the density distribution. The PREM model has polynomial representation of the wavespeeds and density in terms of radius, and ak135 uses piecewise linear segments in radius. The existence of the relation (4) is therefore unexpected, and not a function of the way the models are represented.

It is therefore appropriate to consider (4) as a pragmatic, approximate, representation of the relation of the shear and adiabatic bulk modulus as a function of pressure. This approach was employed by Kennett (2017) to provide an expression for the shear modulus behaviour as a function of relative volume for the Keane equation of state (Keane, 1954).

The coefficients a and b in Eq. (4) can be specified in a general form. In terms of the initial values of the shear and bulk moduli $\left(\mathrm{G}_{0}, \mathrm{~K}_{0}\right)$ and their pressure derivatives $\left(\mathrm{G}_{0^{\prime}}, \mathrm{K}_{0}{ }^{\prime}\right)$, the linear relation can be expressed as:

$\mathrm{G}=\left(\frac{\mathrm{G}_{0}}{\mathrm{~K}_{0}}\right) \mathrm{K}-\left[\left(\frac{\mathrm{G}_{0}}{\mathrm{~K}_{0}}\right) \mathrm{K}_{0}^{\prime}-\mathrm{G}_{0}^{\prime}\right] \mathrm{p}$.

With this general expression it is possible to generate accompanying shear relations for a range of equations of state, as shown in the Appendix. The best-fit lines of regression shown in Fig. 1 correspond very well with this general relation, using tabulated values of the material parameters (Stixrude and Lithgow-Bertelloni, 2005).

It is interesting to make a comparison of the relation (6) and the third-order Birch-Murnaghan formulation that is frequently employed in specifications of material behaviour over moderate pressure ranges. This equation of state is based on a power series expansion of the strain energy in terms of Eulerian strain, which leads to a direct expression for both bulk and shear modulus (Stixrude and Lithgow-Bertelloni, 2005).
In terms of compressive strain

$f=\left[\left(\frac{\rho}{\rho_{0}}\right)^{2 / 3}-1\right]$,

the pressure is given by

$p=(1+2 f)^{5 / 2}\left[3 K_{0} f+\frac{9}{2} K_{0}\left(K_{0}^{\prime}-4\right) f^{2}+\ldots\right]$.

The elastic moduli for the Birch-Murnaghan system can be extracted from an expansion about the hydrostatic state as:

$\mathrm{K}=(1+2 \mathrm{f})^{5 / 2}\left[\mathrm{~K}_{0}+\mathrm{K}_{0}\left(3 \mathrm{~K}_{0}^{\prime}-5\right) \mathrm{f}+\frac{27}{2} \mathrm{~K}_{0}\left(\mathrm{~K}_{0}^{\prime}-4\right) \mathrm{f}^{2}+\ldots\right]$,

G

$$
\begin{aligned}
= & (1+2 \mathrm{f})^{5 / 2}\left[\mathrm{G}_{0}+\left(3 \mathrm{~K}_{0} \mathrm{G}_{0}^{\prime}-5 \mathrm{G}_{0}\right) \mathrm{f}+\left(6 \mathrm{~K}_{0} \mathrm{G}_{0}^{\prime}-24 \mathrm{~K}_{0}-14 \mathrm{G}_{0}+\frac{9}{2} \mathrm{~K}_{0} \mathrm{~K}_{0}^{\prime}\right)\right. \\
& \left.\mathrm{f}^{2}+\ldots\right] .
\end{aligned}
$$

Whereas, the expression for the shear modulus obtained by combining bulk modulus and pressure using (6) is

$$
\begin{aligned}
\widehat{\mathrm{G}}=(1+ & 2 \mathrm{f})^{5 / 2}\left[\mathrm{G}_{0}+\left(3 \mathrm{~K}_{0} \mathrm{G}_{0}^{\prime}-5 \mathrm{G}_{0}\right) \mathrm{f}\right. \\
& \left.+\frac{9}{2}\left\{7 \mathrm{G}_{0} \mathrm{~K}_{0}^{\prime}-4 \mathrm{~K}_{0} \mathrm{G}_{0}^{\prime}-12 \mathrm{G}_{0}+\mathrm{K}_{0}^{\prime}\left(\mathrm{G}_{0}^{\prime} \mathrm{K}_{0}^{\prime}-\mathrm{G}_{0} \mathrm{~K}_{0}^{\prime}\right)\right\} \mathrm{f}^{2}+\ldots\right] .
\end{aligned}
$$

The expressions (10) and (11) for the shear modulus agree as far as the first term in $\mathrm{f}$, but show rather different forms for the $\mathrm{f}^{2}$ term, and so will diverge for increasing compressional strain $f$ and hence pressure. A similar relation holds for general equations of the Birch-Murnaghan type, using other forms of strain measure, as sketched in Appendix B.

The effect can be well illustrated using the material parameters for $\mathrm{MgO}$

$\mathrm{K}_{0}=162.47 \mathrm{GPa}, \quad \mathrm{K}_{0}^{\prime}=3.97, \quad \mathrm{G}_{0}=131.86 \mathrm{GPa}, \quad \mathrm{G}_{0}^{\prime}=2.35$.

In Fig. 3(a) we compare different estimates for the shear modulus behaviour of $\mathrm{MgO}$ as a function of pressure, using plots of $\mathrm{G} / \mathrm{K}$ against $\mathrm{p} / \mathrm{K}$. We compare the results from the full third-order Birch-Murnaghan Eqs. (8)-(10), marked as BM3, with the representation (11) based on the linear relation (6) marked as L. There are substantial differences in the behaviour with the full Birch-Murnaghan results producing a significantly lower shear modulus. We also show the result where the 

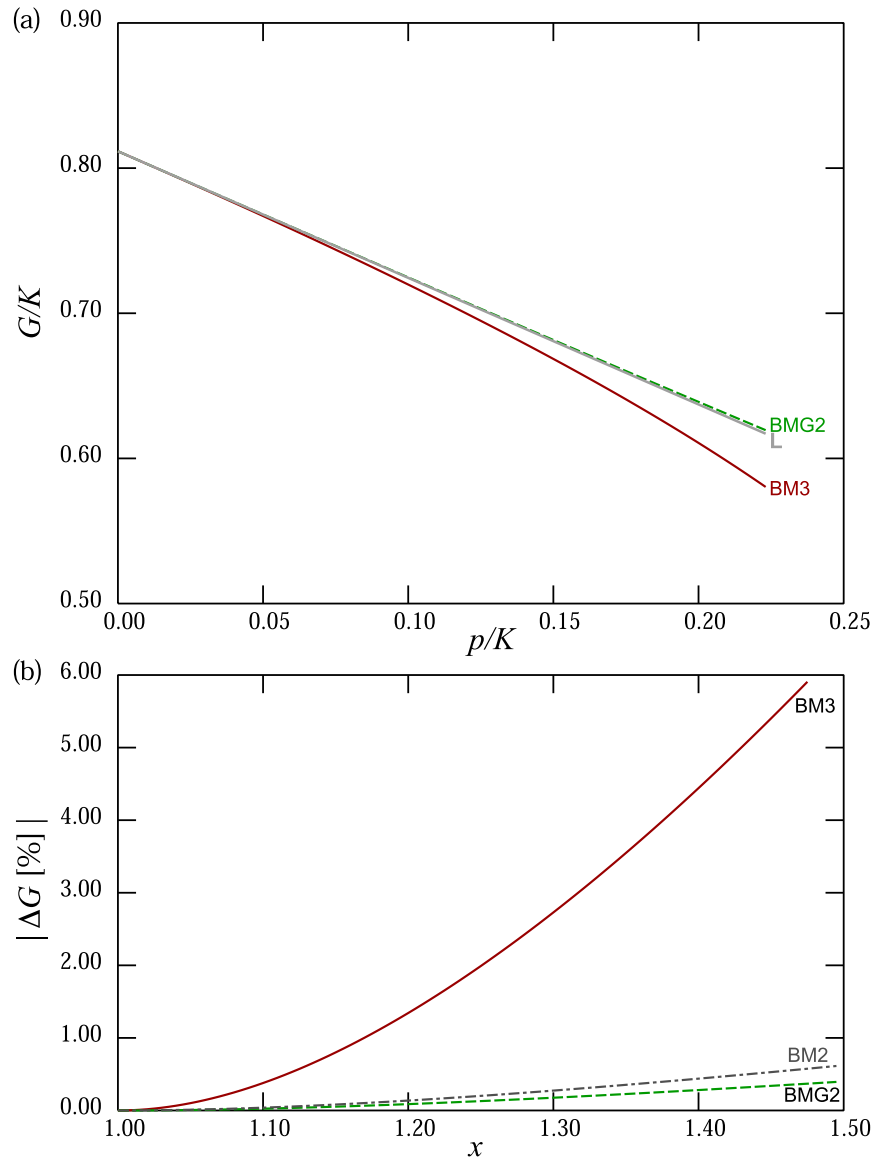

Fig. 3. (a) Deviation of the shear modulus for $\mathrm{MgO}$ from the Birch-Murnaghan third-order equations BM3, from the estimate made using the linear relation (11) marked as $\mathrm{L}$, as a function of $\mathrm{p} / \mathrm{K}$. The result for the expansion of the shear modulus truncated at second order BMG2 is also indicated. (b) The relative deviation in the estimates for $G$ as a function of $x=\rho / \rho_{0}$. The reference is the linear relation (6) applied to the Birch-Murnaghan third-order pressure and bulk-modulus. The deviations are noticeable for the third-order shear modulus BM3, but much less when the shear modulus expansion is truncated BMG2. The difference with using only second-order contributions BM2 is also shown.

expression (10) for the shear modulus is truncated after the $\mathrm{f}$ term, marked as BMG2. This truncated form was employed by Davies and Dziewonski (1975), but as pointed out by Stixrude and LithgowBertelloni (2005) is not fully thermodynamically consistent with the expressions for pressure and bulk modulus. Nevertheless, as can be seen from Fig. 3(a), the truncated expansion gives a very close fit to the prediction from the linear relation (6). The comparisons made by Melinger-Cohen and Jeanloz (2019) indicate that the choice between the thermodynamically consistent and truncated forms is not straightforward, and may well depend on the material being studied.

The absolute values of the deviations of the shear modulus estimates from the linear prediction (11) as a function of the density ratio $x=\rho$ / $\rho_{0}$, are shown in Fig. 3(b). For conditions equivalent to the base of the mantle the differences between the full third-order Birch-Murnaghan results BM3 and the linear prediction can exceed 5\%. Even for $10 \%$ density increase the deviations are of the order of $0.3 \%$, and so beginning to become noticeable. The truncation of the shear modulus to second order BMG2, i.e., terms just in f, is much more consistent with the linear relation. The deviations are less than $0.3 \%$ for the highest pressures and volumetric strain. If the Birch-Murnaghan moduli taken to the term in $\mathrm{f}$ are used throughout (BM2) the deviation from the thirdorder linear prediction are slightly larger. This case will plot directly on the line L in Fig. 3(a). This (BM2) representation was used recently by Gréaux et al. (2019) in a study of the transition zone and uppermost lower mantle.

For $\mathrm{MgO}$ the value of $\mathrm{K}_{0}{ }^{\prime}$ of 3.97 is close to 4 and so the $\mathrm{f}^{2}$ terms in pressure and bulk modulus have a muted effect, because of the presence of the factor $\left(\mathrm{K}_{0^{\prime}}-4\right)$. Stronger deviations between the BirchMurnaghan third-order results and the linear projection are produced when the $\mathrm{K}_{0}{ }^{\prime}$ contributions have greater influence.

As noted above, radial seismological models conform closely to a linear relation between shear modulus, bulk modulus and pressure as in (4). The significant deviation of the third-order Birch-Murnaghan results from this relation may help to explain the difficulties encountered by authors who have tried to fit both bulk and shear moduli for the lower mantle using such representations (e.g. Piazzoni et al., 2007). Commonly one or other of the moduli can be fit, but not both. The same tendency can be seen in inversions for physical parameters for specific materials. Kennett and Jackson (2009) have undertaken a simultaneous inversion for $\mathrm{MgO}$ exploiting a wide range of datasets with the thirdorder Birch-Murnaghan formulation of Stixrude and Lithgow-Bertelloni (2005). Their optimal model for $\mathrm{MgO}$ is compared with the data in Fig. 4 of Kennett and Jackson (2009). This model lies slightly above the values for adiabatic bulk modulus $\mathrm{K}_{\mathrm{S}}$ at higher pressures, but ties directly to those for shear modulus G. Similar behaviour is present in the third-order fits to $\mathrm{K}, \mathrm{G}$ for ab initio calculations for $\mathrm{MgSiO}_{3}$ presented in Fig. 1 of Stixrude and Lithgow-Bertelloni (2005).

The differences between the Birch-Murnaghan results and the linear relation for shear modulus are significant, but the choice between them will depend on the nature of the data being represented. Where an alternative choice has been made for fitting bulk modulus data (e.g. Sakai et al., 2016), the use of a shear modulus representation derived from the linear relation (6) allows the full behaviour to be captured.

\section{Approach to infinite pressure}

Most forms of equations of state are parameterised in terms of the properties of an initial state commonly that with zero pressure, even where this may not be physically attainable for the material being considered. This means that the equations provide a means of extrapolating to higher compression and pressure with a specified functional form. Over a moderate span of pressures differences between different choices of equations remain small, but can become substantial at high pressures for the same specified initial conditions, e.g., Fig. 2 of Kennett (2017). The inclusion of high pressure constraints in the estimation of physical parameters can improve the situation (Kennett and Jackson, 2009), since then the equation of state can be regarded as an interpolant rather than just an extrapolant.

The range of pressures in the Earth (up to $\sim 360 \mathrm{GPa}$ ) is such that only a moderate range of normalised pressure $\mathrm{p} / \mathrm{K}$ is sampled. The extension of constitutive equations to much higher pressures means that the extrapolant may miss aspects of the true behaviour of the materials, e.g., very high pressure phase transitions in $\mathrm{MgO}$ (McWilliams et al., 2012).

Nevertheless, in the absence of direct measurements or computed properties, a state of infinite pressure is often invoked as a limit, under the assumption that the resulting plasma state of matter has simple properties. Models of a single-component plasma, with a lattice of ions in a uniform neutralizing background of electrons, appropriate to elemental behaviour suggest that as the pressure $\bar{p} \rightarrow \infty$, it has the density dependence $\overline{\mathrm{p}} \sim \rho^{5 / 3}$. The bulk modulus for the single-component plasma model is given by $\overline{\mathrm{K}}=\rho \partial \overline{\mathrm{p}} / \partial \rho=\frac{5}{3} \overline{\mathrm{p}}$ and so $\overline{\mathrm{K}}_{\infty}^{\prime}=5 / 3$, as assumed by Holzapfel (1998). The shear contribution for this plasma state is expected to come from the ionic component (Burakovsky et al., 2004) so that $\overline{\mathrm{G}} \sim \rho^{4 / 3}$ and $\overline{\mathrm{G}} / \overline{\mathrm{K}} \rightarrow 0$ as $\overline{\mathrm{p}} \rightarrow \infty$. This shear property led Burakovsky et al. (2004) to suggest that the relation between shear modulus G, bulk modulus $\mathrm{K}$ and pressure $\mathrm{p}$ should take the form

$\mathrm{G}=\left(\frac{\mathrm{G}}{\mathrm{K}}\right)_{0}[\mathrm{~K}-\varpi(\mathrm{p}) \mathrm{p}]$. 
where

$\varpi(\mathrm{p})=\frac{\mathrm{K}}{\mathrm{p}}\left[1-\left(\frac{\mathrm{K}}{\mathrm{G}}\right)_{0}\left(\frac{\mathrm{G}}{\mathrm{K}}\right)\right]$

is a slowly varying monotonic function, with

$\varpi(0)=\left[\mathrm{K}_{0}^{\prime}-\left(\frac{\mathrm{K}}{\mathrm{G}}\right)_{0} \mathrm{G}_{0}^{\prime}\right], \quad \varpi(\overline{\mathrm{p}}) \rightarrow \frac{5}{3} \quad$ as $\quad \overline{\mathrm{p}} \rightarrow \infty$.

Stacey and Davis (2004) have taken a different position and argue that for materials relevant to the Earth, $\mathrm{K}_{\infty}^{\prime}$ depends on the material and, on thermodynamic grounds, $K_{\infty}^{\prime}>5 / 3$. From earlier arguments by Keane (1954), a likely upper bound is provided by $\mathrm{K}_{\infty}^{\prime}<\mathrm{K}_{0}^{\prime}-1$. Stacey and Davis (2004) also suggest that $(\mathrm{G} / \mathrm{K})_{\infty}$ has a finite value and is a specific material property. As for the zero pressure properties, the extrapolation to infinite pressure is associated with a particular material state, rather than corresponding directly to the effects of extreme pressure.

Although the linear relation for shear modulus would appear to be satisfactory over the pressure range up to $360 \mathrm{GPa}$ encountered in the Earth (p/K 0.25), the arguments of Burakovsky et al. (2004) would suggest that deviations can be expected at extreme pressures. Thus the properties of $\varpi(\mathrm{p})$ in (15) should be modified to

$\varpi(\overline{\mathrm{p}}) \rightarrow \mathrm{K}_{\infty}^{\prime}\left[1-\left(\frac{\mathrm{K}}{\mathrm{G}}\right)_{0}\left(\frac{\mathrm{G}}{\mathrm{K}}\right)_{\infty}\right]$ as $\mathrm{p} \rightarrow \infty$,

which would imply a direct link between the apparent compression and shear properties at infinite pressure.

In a representation of an equation of state in terms of volumetric strain $x=\rho / \rho_{0}$, the value of $\mathrm{K}_{\infty}^{\prime}$ is given by the exponent of the largest power of $x$. Although equations of state are usually selected for their suitability for fitting data, Stacey and Davis (2004) insist that that they must satisfy the infinite pressure condition $\mathrm{K}_{\infty}^{\prime}>5 / 3$. Certainly this would seem to be appropriate for application to the inner core, but may not be necessary elsewhere in the Earth. The Keane (1954) equation of state includes $\mathrm{K}_{\infty}^{\prime}$ as a natural parameter and is very suitable for deep Earth conditions. However, attempts to adjust other equations of state by modifying the leading exponent to meet this infinite pressure condition run the risk of producing a state of negative pressure within the range of reasonable compressions, and may not correspond to a sensible strain energy function.

\section{Discussion and conclusions}

A general formulation for the representation of shear modulus behaviour under pressure can be provided by including a deviatoric term in the representation of strain energy. The functional form of the shear modulus behaviour can be derived from the semi-empirical linear relation between shear modulus, bulk modulus and pressure that is well satisfied by experimental and computational results, and current Earth models. The specific behaviour is controlled by the zero pressure values of the shear and bulk modulus $\left(\mathrm{G}_{0}, \mathrm{~K}_{0}\right)$ and their pressure derivatives $\left(\mathrm{G}_{0}{ }^{\prime}, \mathrm{K}_{0}{ }^{\prime}\right)$. In this way shear modulus forms can be provided for commonly used equations of state. No adiabatic corrections have to be applied to the shear modulus.

This approach provides an alternative to the use of the BirchMurnaghan expansion based on powers of Eulerian strain, which although thermodynamically consistent gives results at third-order that deviate significantly from the linear relation between $\mathrm{K} / \mathrm{p}$ and $\mathrm{G} / \mathrm{p}$.

For pressures up to $25 \mathrm{GPa}$, corresponding to the full upper mantle, there is little difference between the results with different equation of state formulations, and this is the domain for which the conventional Birch-Murnaghan development is most appropriate. The logarithmic equation-of-state of Poirier and Tarantola (1998) can be carried well into the lower mantle, at least to $35 \mathrm{GPa}$, and is now accompanied by a shear modulus expression.

For lower mantle conditions the Vinet-Rydberg-Morse form (Vinet et al., 1987) can be effective to at least $100 \mathrm{GPa}$ (e.g., Kennett, 2017), but for the zone near the core-mantle boundary Sakai et al. (2016) favour the application of the Keane EOS (Keane, 1954) or AP2 (Holzapfel, 1998). These two representations take into account expected behaviour under extreme pressure conditions, and are now provided with accompanying shear modulus forms.

Although Irving et al. (2018) have successfully used the Vinet and the third-order Birch-Murnagan equations in inversion of normal mode results for compressional wavespeed in the core, neither formulation has appropriate behaviour for very high pressures. In the core regime the Keane formulation is to be preferred. The nearly equivalent reciprocal K' approach (Stacey and Davis, 2004; Stacey, 2019) is formulated in terms of pressure rather than density ratio, and so is less suited to direct comparison with experimental results.

\section{Author statement}

The entire paper is work of the sole author B.L.N. Kennett.

\section{Declaration of competing interest}

The authors declare that they have no known competing financial interests or personal relationships that could have appeared to influence the work reported in this paper.

\section{Appendix A. Cold equations of state and associated shear modulus}

We consider commonly used equations of state (EOS), without consideration of the influence of temperature. A number of different formulations have been used to fit experimental data on material properties at high pressure, and can be written in terms of the density ratio $\mathrm{x}=\rho / \rho_{0}=\mathrm{V}_{0} /$ $\mathrm{V}=\mathrm{J}^{-1}$. This 'cold' part of equations of state provides a specification of the pressure $p$ as a function of volume $\mathrm{p}(\mathrm{V})$ or, equivalently, density ratio $\mathrm{p}$ $(\mathrm{x})$. The bulk modulus $\mathrm{K}$ can be extracted from the expressions for the pressure in the EOS from $\mathrm{K}=-\mathrm{V}(\partial \mathrm{p} / \partial \mathrm{V})_{\mathrm{T}}=\mathrm{x}(\partial \mathrm{p} / \partial \mathrm{x})$. A further differentiation extracts the pressure derivative $\mathrm{K}^{\prime}=(\partial \mathrm{K} / \partial \mathrm{p})_{\mathrm{T}}=\mathrm{x}(\partial \mathrm{K} / \partial \mathrm{x}) / \mathrm{K}$.

For each of the major styles of EOS employed in deep Earth studies, we specify the pressure $\mathrm{p}$ and bulk modulus $\mathrm{K}$ dependence of volumetric strain $\mathrm{x}$, together with the form for the shear modulus derived from Eq. (6). We use a parametrisation in terms of the moduli $\mathrm{K}_{0}$, $\mathrm{G}_{0}$ and their pressure derivatives $\mathrm{K}_{0}{ }^{\prime}, \mathrm{G}_{0}{ }^{\prime}$ at ambient conditions.

Each form of EOS should be regarded as a parametric representation of behaviour, and thus when different expressions are used to fit the same experimental data the values obtained for $\mathrm{K}_{0}, \mathrm{G}_{0}, \mathrm{~K}_{0}{ }^{\prime}, \mathrm{G}_{0}{ }^{\prime}$ will be similar but not identical (see, e.g., Sakai et al., 2016).

\section{A.1. Poirier and Tarantola - logarithmic EOS}

Poirier and Tarantola (1998) use an expansion of the strain energy in terms of logarithmic strain, which gives a more rapid convergence than the Birch-Murnaghan expansion in terms of Eulerian strain. To second order, the pressure is 
$\mathrm{p}=\mathrm{K}_{0} \mathrm{x}\left[\ln x+\frac{1}{2}\left(\mathrm{~K}_{0}^{\prime}-2\right)(\ln \mathrm{x})^{2}\right]$.

To the same order the bulk modulus is

$\mathrm{K}=\mathrm{K}_{0} \mathrm{x}\left[1+\left(\mathrm{K}_{0}^{\prime}-1\right) \ln \mathrm{x}+\frac{1}{2}\left(\mathrm{~K}_{0}^{\prime}-2\right)(\ln \mathrm{x})^{2}\right]$.

The associated representation for the shear modulus $\mathrm{G}$ takes the form:

$\mathrm{G}=\mathrm{x}\left[\mathrm{G}_{0}+\left(\mathrm{K}_{0} \mathrm{G}_{0}^{\prime}-1\right) \ln \mathrm{x}+\frac{1}{2}\left(\mathrm{~K}_{0}^{\prime}-2\right)\left[\mathrm{K}_{0} \mathrm{G}_{0}^{\prime}-\mathrm{G}_{0}\left(\mathrm{~K}_{0}^{\prime}-1\right)\right](\ln \mathrm{x})^{2}\right]$.

\section{A.2. Vinet-Rydberg-Morse EOS}

The Vinet-Rydberg-Morse EOS is based on an atomic force model. Earlier work by Rydberg and Morse was reformulated independently by Vinet et al. (1987). Pressure is represented as

$\mathrm{p}=3 \mathrm{~K}_{0} \mathrm{x}^{2 / 3}\left(1-\mathrm{x}^{-1 / 3}\right) \exp \left\{\zeta\left[1-\mathrm{x}^{-1 / 3}\right]\right\}$,

with $\zeta=\frac{3}{2}\left(\mathrm{~K}_{0}^{\prime}-1\right)$. The bulk modulus is

$\mathrm{K}=\mathrm{K}_{0} \mathrm{x}^{2 / 3}\left[2+(\zeta-1) \mathrm{x}^{-1 / 3}-\zeta \mathrm{x}^{-2 / 3}\right] \exp \left\{\zeta\left[1-\mathrm{x}^{-1 / 3}\right]\right\}$,

and the associated shear modulus is

$\mathrm{G}=\mathrm{x}^{2 / 3}\left[\mathrm{G}_{0}\left[2+(\zeta-1) \mathrm{x}^{-1 / 3}-\zeta \mathrm{x}^{-2 / 3}\right]+\left(\mathrm{K}_{0} \mathrm{G}_{0}^{\prime}-\mathrm{G}_{0} \mathrm{~K}_{0}^{\prime}\right)\left(1-\mathrm{x}^{-1 / 3}\right)\right] \exp \left\{\zeta\left[1-\mathrm{x}^{-1 / 3}\right]\right\}$.

\section{A.3. Keane EOS}

The Keane (1954) EOS links to properties at (nominal) infinite pressure, introducing the additional parameter $\mathrm{K}_{\infty}{ }^{\prime}=\lim _{\mathrm{p} \rightarrow \infty}[\mathrm{E} / \mathrm{p}]$ with a lower bound of 5/3 from thermodynamic arguments (Stacey and Davis, 2004).

The pressure is given by

$\mathrm{p}=\mathrm{K}_{0}\left[\frac{\mathrm{K}_{0}^{\prime}}{\mathrm{K}_{\infty}^{\prime 2}}\left[\mathrm{x}^{\mathrm{K}_{\infty}^{\prime}}-1\right]-\left(\frac{\mathrm{K}_{0}^{\prime}}{\mathrm{K}_{\infty}^{\prime}}-1\right) \ln \mathrm{x}\right]$.

The Keane representation of the bulk modulus has a rather simple form,

$\mathrm{K}=\mathrm{K}_{0}\left[1+\frac{\mathrm{K}_{0}^{\prime}}{\mathrm{K}_{\infty}^{\prime}}\left(\mathrm{x}_{\infty}^{\mathrm{K}_{\infty}^{\prime}}-1\right)\right]$

The associated shear modulus is

$\mathrm{G}=\mathrm{G}_{0}\left[1+\frac{\mathrm{K}_{0}^{\prime}}{\mathrm{K}_{\infty}^{\prime}}\left(\mathrm{x}^{\mathrm{K}_{\infty}^{\prime}}-1\right)\right]+\left(\mathrm{K}_{0} \mathrm{G}_{0}^{\prime}-\mathrm{G}_{0} \mathrm{~K}_{0}^{\prime}\right)\left[\frac{\mathrm{K}_{0}^{\prime}}{\mathrm{K}_{\infty}^{\prime 2}}\left[\mathrm{x}^{\mathrm{K}_{\infty}^{\prime}}-1\right]-\left(\frac{\mathrm{K}_{0}^{\prime}}{\mathrm{K}_{\infty}^{\prime}}-1\right) \ln \mathrm{x}\right]$.

An alternative, equivalent form for the shear modulus was provided by Kennett (2017).

\section{A.4. Holzapfel AP2 EOS}

The AP2 EOS (Holzapfel, 1998) was developed for regular, elemental solids under strong compression. It has been successfully used by Sakai et al. (2016) in a study of post-perovskite. The pressure is given by

$\mathrm{p}=3 \mathrm{~K}_{0} \mathrm{x}^{5 / 3}\left(1-\mathrm{x}^{-1 / 3}\right)\left[1+\mathrm{c}_{2} \mathrm{x}^{-1 / 3}\left(1-\mathrm{x}^{-1 / 3}\right)\right] \exp \left[\mathrm{c}_{0}\left(1-\mathrm{x}^{-1 / 3}\right)\right]$

with $\mathrm{c}_{2}=\frac{3}{2}\left(\mathrm{~K}_{0}^{\prime}-3\right)-\mathrm{c}_{0}$, and $\mathrm{c}_{0}=-\ln \left(3 \mathrm{~K}_{0} / \mathrm{p}_{\mathrm{F}}\right)$, where $\mathrm{p}_{\mathrm{F}}=\mathrm{a}_{\mathrm{F}}\left(\mathrm{Z} / \mathrm{V}_{0}{ }^{5 / 3}\right)$ where $\mathrm{Z}$ stands for the total electron number in the crystallographic unit cell with the corresponding volume $\mathrm{V}_{0}$. The constant $\mathrm{a}_{\mathrm{F}}=0.02336965 \mathrm{GPa} \mathrm{nm}^{5}$.

The expression for the bulk modulus takes the form:

$\begin{aligned} \mathrm{K}= & \mathrm{K}_{0} \mathrm{x}\left\{\left[\left(5 \mathrm{x}^{2 / 3}-4 \mathrm{x}^{1 / 3}\right)+\mathrm{c}_{0}\left(\mathrm{x}^{1 / 3}-1\right)\right]\left[1+\mathrm{c}_{2} \mathrm{x}^{-1 / 3}\left(1-\mathrm{x}^{-1 / 3}\right)\right]\right\} \mathrm{E} \\ & +\mathrm{K}_{0} \mathrm{x}\left\{\mathrm{c}_{2}\left(1-\mathrm{x}^{-1 / 3}\right)\left(2-\mathrm{x}^{1 / 3}\right)\right\} E,\end{aligned}$

The shear modulus can be constructed from (6),

$\mathrm{G}=\mathrm{G}_{0}\left[\frac{\mathrm{K}}{\mathrm{K}_{0}}\right]+\left(\mathrm{K}_{0} \mathrm{G}_{0}^{\prime}-\mathrm{G}_{0} \mathrm{~K}_{0}^{\prime}\right)\left[\frac{\mathrm{p}}{\mathrm{K}_{0}}\right]$

\section{Appendix B. Strain energy expansions in terms of generalised strain measures}

Melinger-Cohen and Jeanloz (2019) have presented a full treatment of the expansion of strain energy in terms of quadratic strain measures (both Eulerian and Lagrangian) for the case of cubic materials, and Katsura and Tange (2019) have investigated the use of alternative strain measures for the bulk modulus for isotropic materials.

A corresponding development can be made for the full family of Seth-Hill strain measures (3). For each Seth-Hill strain, except $q=0$, we 
introduce the compressive strain measure

$\mathrm{f}_{\mathrm{q}}=\frac{1}{\mathrm{q}}\left(1-\mathrm{J}^{\mathrm{q} / 3}\right)$.

Thus the Eulerian compressive strain used in the Birch-Murnaghan development is $\mathrm{f}_{-2}$.

For an isotropic material under compression, the strain energy $W$ can be expanded in powers of $\mathrm{f}_{\mathrm{q}}$,

$W=W_{0}+\mathrm{a}_{2} \mathrm{f}_{\mathrm{q}}^{2}+\mathrm{a}_{3} \mathrm{f}_{\mathrm{q}}^{3}+\ldots$

The coefficient of the linear term in $\mathrm{f}_{\mathrm{q}}$ has to vanish because the initial state is at zero pressure. For this form of strain energy, pressure is given by

$\mathrm{p}=\left(1-\mathrm{qf}_{\mathrm{q}}\right)^{1-3 / \mathrm{q}}\left[3 \mathrm{~K}_{0} \mathrm{f}_{\mathrm{q}}+\frac{9}{2} \mathrm{~K}_{0}\left(\mathrm{~K}_{0}^{\prime}-2+q\right) \mathrm{f}_{\mathrm{q}}^{2}+\ldots\right]$.

The associated bulk and shear moduli are

$\mathrm{K}=\left(1-\mathrm{qf}_{\mathrm{q}}\right)^{1-3 / \mathrm{q}}\left[\mathrm{K}_{0}+\mathrm{K}_{0}\left[3 \mathrm{~K}_{0}^{\prime}-(3-\mathrm{q})\right] \mathrm{f}_{\mathrm{q}}+\ldots\right]$,

$\mathrm{G}=\left(1-\mathrm{qf}_{\mathrm{q}}\right)^{1-3 / \mathrm{q}}\left[\mathrm{G}_{0}+\left[3 \mathrm{~K}_{0} \mathrm{G}_{0}^{\prime}-(3-\mathrm{q}) \mathrm{G}_{0}\right] \mathrm{f}_{\mathrm{q}}+\ldots\right]$.

For all non-zero values of $q$ the bulk and shear moduli satisfy the linear relation (6) with pressure to order $\mathrm{f}_{\mathrm{q}}$, but not to higher orders. The discrepancies are larger for Lagrangian style measures with $\mathrm{q}>0$, than for the Eulerian style $(\mathrm{q}<0)$, again reinforcing the preference for $\mathrm{q}<0$ in a state of compression.

\section{References}

Beex, L.A.A., 2019. Fusing the Seth-Hill strain tensors to fit compressible elastic material responses in the nonlinear regime. Int. J. Mech. Sci. 163, 105072.

Burakovsky, L., Preston, D.L., Wang, Y., 2004. Cold shear modulus and Grüneisen parameter at all densities. Solid State Commun. 132, 151-156.

Davies, G.F., Dziewonski, A.M., 1975. Homogeneity and constitution of Earth's lower mantle and outer core. Phys. Earth Planet. Inter. 10, 336-343.

Dziewoński, A.M., Anderson, D.L., 1981. Preliminary reference earth model. Phys. Earth Planet. Inter. 25, 297-356.

Elcombe, M.H., 1967. Some aspects of the lattice dynamics of quartz. Proc. Phys. Soc. London 91, 947-958.

Falzone, A.J., Stacey, F.D., 1980. Second order elasticity theory: explanation for the high Poisson's ratio of the inner core. Phys. Earth Planet. Inter. 21, 371-377.

Gréaux, S., Irifune, T., Higo, Y., Tange, Y., Arimoto, T., Liu, Z, Yamada, A., 2019. Sound velocity of $\mathrm{CaSiO} 3$ perovskite suggests the presence of basaltic crust in the Earth's lower mantle. Nature 565, 218-221.

Hill, R., 1968. On constitutive inequalities for simple materials-I. J. Mech. Phys. Solids $16,229-242$.

Holzapfel, W.B., 1998. Equations of state for solids under strong compression. High Press. Res. 16, 81-126.

Irving, J.C.E., Cottaar, S., Lekić, V., 2018. Seismically determined elastic parameters for Earth's outer core. Sci. Adv. 4 (6), eaar2538.

Jackson, I., Niesler, H., 1982. The elasticity of periclase to 3GPa and some geophysica implications. In: Akimoto, S., Manghnani, M.H. (Eds.), High-pressure Research in Geophysics. Centre of Academic Publications, Japan, pp. 93-113.

Karki, B.B., Stixrude, L., Clark, S.J., Warren, M.C., Ackland, G.J., Crain, J., 1997. Structure and elasticity of $\mathrm{MgO}$ at high pressure. Am. Mineral. 82, 51-60.

Katsura, T., Tange, Y, 2019. A simple derivation of the Birch-Murnaghan equations of state (EOSs) and comparison with EOSs derived from other definitions of finite strain. Minerals 9, 745. https://doi.org/10.3390/min9120745.

Keane, A., 1954. An investigation of finite strain in an isotropic material subjected to hydrostatic pressure and its seismological applications. Austr. J. Phys. 7, 322-333.

Kennett, B.L.N., 2017. Towards constitutive equations for the deep Earth. Phys. Earth Planet. Inter. 270, 40-45.

Kennett, B.L.N., Jackson, I., 2009. Optimal equations of state for mantle minerals from simultaneous non-linear inversion of multiple datasets. Phys. Earth Planet. Inter. 176, 98-108.

Kennett, B.L.N., Engdahl, E.R., Buland, R., 1995. Constraints on seismic velocities in the Earth from travel times. Geophys. J. Int. 122, 108-124.

Kieffer, S.W., 1979. Thermodynamics and lattice vibrations of minerals: 3. Lattice dynamics and an approximation for minerals with application to simple substances and framework silicates. Rev. Geophys. Space Phys. 17, 35-59.

Kono, Y., Irifune, T., Higo, Y., Inoue, T., Barnhoorn, A., 2010. P-V-T relation of MgO derived by simultaneous elastic wave velocity and in situ X-ray measurements: a new pressure scale for the mantle transition region. Phys. Earth Planet. Inter. 183, 196-211.

Marquardt, H., Speziale, S., Reichmann, H.J., Frost, D.J., Schilling, F.R., 2009. Single crystal elasticity of $\left(\mathrm{Mg}_{0.9} \mathrm{Fe}_{0.1}\right) \mathrm{O}$ to $81 \mathrm{GPa}$. Earth Planet. Sci. Lett. 287, 345-352.

McWilliams, R.S., Spaulding, D.K., Eggert, J.H., Celliers, P.M., Hicks, D.G., Smith, R.F. Collins, G.W., Jeanloz, R., 2012. Phase transformations and metallization of magnesium oxide at high pressure and temperature. Science 338, 1330-1333.

Melinger-Cohen, A., Jeanloz, R., 2019. Finite strain analysis of shear and compressional wave velocities. J. Geophys. Res.: Solid Earth 124, 11 651-11 677.

Montagner, J.-P., Kennett, B.L.N., 1996. How to reconcile body-wave and normal-mode reference earth models? Geophys. J. Int. 125, 229-248.

Murakami, M., Ohishi, Y., Hirao, N., Hirose, K., 2009. Elasticity of MgO to 130 GPa: implications for lower mantle mineralogy. Earth Planet. Sci. Lett. 277, 123-129.

Piazzoni, A.S., Steinle-Neumann, G., Bunge, H.P., Dolejš, D., 2007. A mineralogical model for density and elasticity of the Earth's mantle. Geochem. Geophys. Geosyst. 8 (11), Q11010. https://doi.org/10.1029/2007GC001697.

Poirier, J.-P., Tarantola, A., 1998. A logarithmic equation of state. Phys. Earth Planet. Inter 109, 1-8.

Sakai, T., Dekura, H., Hirao, N., 2016. Experimental and theoretical thermal equations of state of $\mathrm{MgSiO}_{3}$ post-perovskite at multi-megabar pressures. Sci. Rep. 6, 22652. https://doi.org/10.1038/srep22652.

Seth, B.R., 1964. Generalized strain measure with application to physical problems. In: Reiner, M., Abir, D. (Eds.), Second-order Effects in Elasticity, Plasticity and Fluid Dynamics. Pergamon Press, Oxford, pp. 162-172.

Sinogeikin, S.V., Bass, J.D., 2000. Single-crystal elasticity of pyrope and MgO to $20 \mathrm{GPa}$ by Brillouin scattering in the diamond cell. Phys. Earth Planet. Int. 120, 43-62.

Stacey, F.D., 2019. Equations of state for the deep earth: some fundamental considerations. Minerals 9, 636. https://doi.org/10.3390/min9100636.

Stacey, F.D., Davis, P.M., 2004. High pressure equations of state with applications to the lower mantle and core. Phys. Earth Planet. Inter. 142, 137-184.

Stixrude, L., Lithgow-Bertelloni, C., 2005. Thermodynamics of mantle minerals - I. physical properties. Geophys. J. Int. 162, 610-632.

Stixrude, L., Lithgow-Bertelloni, C., 2011. Thermodynamics of mantle minerals - II. Phase equilibria. Geophys. J. Int. 184, 1180-1213.

Vinet, P., Ferrante, J., Rose, J.H., Smith, J.R., 1987. Compressibility of solids. J. Geophys Res. 92, 9319-9325.

Zha, C.-S., Duffy, T.S., Downs, R.T., Mao, H.-K., Hemley, R.J., 1996. Sound velocity and elasticity of single-crystal forsterite to 16 GPa. J. Geophys. Res. 101, 17,535-17,545.

Zha, C.-S., Mao, H.-K., Hemley, R.J., 2000. Elasticity of $\mathrm{MgO}$ and a primary pressure scale to $55 \mathrm{GPa}$. PNAS 97, 13494-13499.

Zhang, W.-W., Yu, H., Lei, S.-Y., Huang, Q.-A., 2011. Modelling of the elastic properties of crystalline silicon using lattice dynamics. J. Phys. D. Appl. Phys. 44, 335401. https:// doi.org/10.1088/0022-3727/44/33/335401. 